\title{
A genome-wide study of panic disorder suggests the amiloride-sensitive cation channel 1 as a candidate gene
}

\author{
Noomi Gregersen ${ }^{\star 1,2,10}$, Hans A Dahl ${ }^{3,4,10}$, Henriette N Buttenschøn ${ }^{1}$, Mette Nyegaard ${ }^{2,5}$, Anne Hedemand ${ }^{2}$, \\ Thomas D Als ${ }^{1,6}$, August G Wang ${ }^{7}$, Sofus Joensen ${ }^{8}$, David PD Woldbye ${ }^{9}$, Pernille Koefoed ${ }^{9}$, Ann S Kristensen ${ }^{1}$, \\ Torben A Kruse ${ }^{4}$, Anders D Børglum ${ }^{1,2}$ and Ole Mors ${ }^{1}$
}

Panic disorder (PD) is a mental disorder with recurrent panic attacks that occur spontaneously and are not associated to any particular object or situation. There is no consensus on what causes PD. However, it is recognized that PD is influenced by environmental factors, as well as genetic factors. Despite a significant hereditary component, genetic studies have only been modestly successful in identifying genes of importance for the development of PD. In this study, we conducted a genome-wide scan using microsatellite markers and PD patients and control individuals from the isolated population of the Faroe Islands. Subsequently, we conducted a fine mapping, which revealed the amiloride-sensitive cation channel 1 (ACCN1) located on chromosome 17q11.2-q12 as a potential candidate gene for PD. The further analyses of the ACCN1 gene using singlenucleotide polymorphisms (SNPs) revealed significant association with PD in an extended Faroese case-control sample. However, analyses of a larger independent Danish case-control sample yielded no substantial significant association. This suggests that the possible risk alleles associated in the isolated population are not those involved in the development of $\mathrm{PD}$ in a larger outbred population.

European Journal of Human Genetics (2012) 20, 84-90; doi:10.1038/ejhg.2011.148; published online 3 August 2011

Keywords: panic disorder; genome-wide scan; isolated population; association analysis; ACCN1

\section{INTRODUCTION}

Panic disorder (PD) is a common mental disorder in society. ${ }^{1,2}$ It is characterized by recurrent, unprovoked and unpredictable panic attacks, followed by concern of subsequent attacks, resulting in a strong social and functional inhibition. ${ }^{3,4}$ Estimates from family and twin studies ascribe a genetic contribution of approximately $40 \%$ to the disease etiology of the disorder. ${ }^{5-8}$ However, the mechanism underlying $\mathrm{PD}$ is still unknown and presumably involves numerous susceptibility genes with major and/or minor effects. ${ }^{9}, 10$ Furthermore, the possibility of allelic heterogeneity, which most likely will reduce the power of performed studies to detect associated genes, exists. In this context, isolated populations are considered advantageous, as they possess highly beneficial features for diminishing genetic and allelic heterogeneity. ${ }^{11-13}$ In the present study, we use the isolated population of the Faroe Islands to search for susceptibility genes for PD. The population history of this isolate in context of genetics has previously been described. ${ }^{14,15}$ The isolate has formerly been used to locate chromosomal regions associated with other complex disorders, ${ }^{16,17}$ which has lead to identification of genes for schizophrenia and bipolar disorder in larger outbred populations. ${ }^{18-20}$
We present the results from a three-stage genetic investigation of PD (Figure 1). First, we conducted a genome-wide scan on 13 distantly related patients with PD and 43 control individuals from the Faroe Islands. Second, we performed a fine mapping of the chromosome regions observed to be significantly associated with $\mathrm{PD}$, using the same sample as in the genome-wide scan. In this paper, we only report the results from the chromosome $17 \mathrm{q} 11.2-\mathrm{q} 12$ region. Third, the amiloride-sensitive cation channel 1 ( $A C C N 1)$ gene was analysed for association with PD in an extended Faroese case-control sample and in an outbred Danish case-control sample.

\section{MATERIALS AND METHODS}

Subjects and clinical assessment

The Faroese sample. Thirteen patients with $\mathrm{PD}$ and 43 control individuals were included in the genome-wide scan. Recently, additional 18 patients and 119 control individuals were recruited to the Genetic Biobank of the Faroe Islands. All patients were interviewed using the Present State Examination. ${ }^{21}$ The inclusion criteria were PD with or without agoraphobia, according to the ICD-10 diagnostic criteria. ${ }^{3}$ The control individuals were evaluated to be healthy and were matched to

\footnotetext{
${ }^{1}$ Centre for Psychiatric Research, Aarhus University Hospital, Risskov, Aarhus, Denmark; ${ }^{2}$ Department of Human Genetics, Aarhus University, Aarhus, Denmark; ${ }^{3}$ Amplexa Genetics A/S, Odense, Denmark; ${ }^{4}$ Department of Clinical Genetics, Odense University Hospital, Odense, Denmark; ${ }^{5}$ Department of Haematology, Aalborg Hospital Science and Innovation Center AHSIC, Aarhus University Hospital, Aalborg, Denmark; ${ }^{6}$ National Institute of Aquatic Resources, Technical University of Denmark, Lyngby, Denmark; ${ }^{7}$ Department of Psychiatry, HS Amager Hospital, Copenhagen University Hospital, Copenhagen, Denmark; ${ }^{8}$ Department of Psychiatry, National Hospital, Torshavn, Faroe Islands; ${ }^{9}$ Laboratory of Neuropsychiatry, Psychiatric Center Copenhagen, Rigshospitalet and Department of Neuroscience and Pharmacology, University of Copenhagen, Copenhagen, Denmark

*Correspondence: N Gregersen, Centre for Psychiatric Research, Aarhus University Hospital, Skovagervej 2, Risskov 8240, Denmark. Tel: +45 77 8935 62; Fax: +45 778935 99; E-mail: noomigregersen@mac.com

10These authors contributed equally to this work.

Received 19 January 2011; revised 11 May 2011; accepted 24 June 2011; published online 3 August 2011
} 


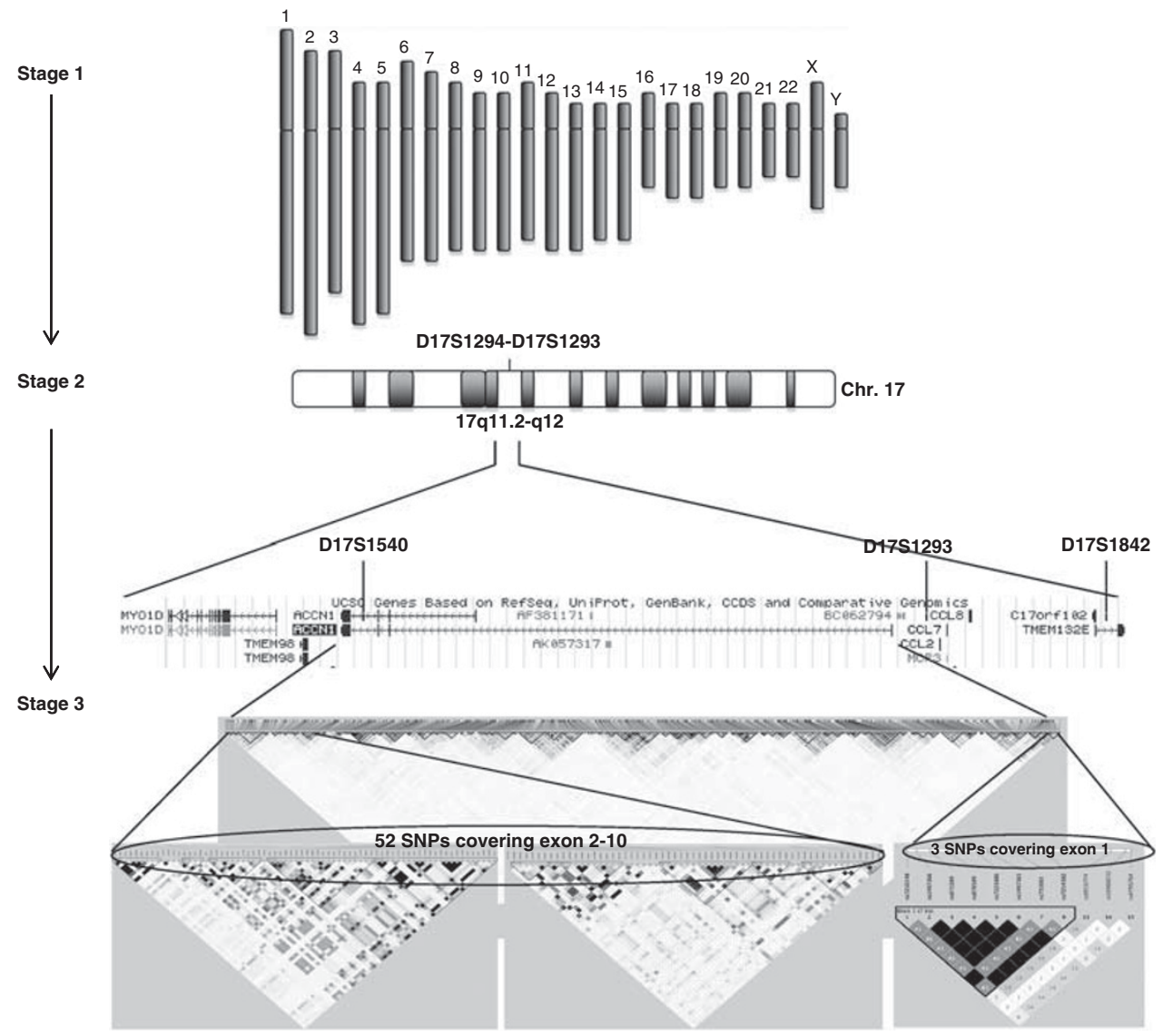

Figure 1 An overview of the study design: in stage 1, we conducted a genome-wide scan, which detected significant association between PD and a twomarker segment (D17S1294-D17S1293) on chromosome 17. In stage 2, we followed up on 17q11.2-q12, which revealed significant association between PD and several markers (D17S1540, D17S1293 and D17S1842) within this region and suggested ACCN1 as a possible candidate gene. In stage 3, we analysed $A C C N 1$ for association with PD, using tag-SNPs.

the cases by ethnicity. A detailed description of the sample has been described in Wang et al. ${ }^{22}$ The extended Faroese sample consisted of 31 PD patients and 162 healthy control individuals.

The Danish sample. The Danish sample consisted of 243 patients with PD and 645 healthy control individuals. The sample was collected from two Danish cohorts, one in the area of Copenhagen ${ }^{23}$ and the other in Jutland. ${ }^{24}$ The patients were diagnosed with PD with or without agoraphobia, according to the ICD-10 diagnostic criteria. ${ }^{3}$ All the patients and controls were of Danish Caucasian origin.

\section{Genotyping}

Stage 1: The genome-wide scan. Thirteen patients with PD and 43 control individuals from the Faroe Islands were genotyped, using approximately 500 microsatellite markers with an average intermarker distance of $5 \mathrm{cM}$ (range $0-14 \mathrm{cM}$ ). Primer sequences were obtained from the Human Genome Database (primer sequences are available on request). DNA fragments were amplified using standard PCR conditions in single or multiplex reactions in a concentration of $6 \mathrm{ng} / \mu \mathrm{l}$ DNA. The PCR fragments were analysed on the ABI 377 genetic analyser (Applied Biosystems, Foster City, CA, USA) and the alleles were analysed using the Genemapper software version 3.7 (Applied Biosystems).

Several chromosomal regions (4p16.1, 17q11.2-q12 and 19p13.2) showed significant association in the genome-wide scan. These regions were submitted to further analyses in order to verify the observation and to further delineate the identical by descended (IBD) status of the regions.

Stage 2: The 17q11.2-q12 region. Given the results, we have, in the present study, chosen to focus on the results from chromosome 17. To verify the finding and to further delineate the IBD status of the 17q11.2-q12 region, the marker density was increased from 20 to 42 microsatellite markers (29 in the $17 \mathrm{q} 11.2$-q12 region), including the 5-HTTLPR repeat in the promoter region of the serotonin transporter (SLC6A4) gene. The study sample and analytic procedures were the same as in the genome-wide scan. Subsequently, the promoter region of the ACCN1 gene was sequenced in 14 individuals using the ABI Big Dye Terminator 3.1 kit (Applied Biosystems). The sequencing revealed seven single-nucleotide polymorphisms (SNPs; rs28936, rs28935, rs28933, rs62068265, rs9916605, rs7214382 and rs2228990), which were genotyped in the complete sample from the genome-wide scan, using the SNaPshot protocol from Applied Biosystems and analysed on an ABI 377 genetic analyser (Applied Biosystems).

Stage 3: Amiloride-sensitive cation channel 1 (ACCN1). A bioinformatic search of the chromosome segment comprising the 17q11.2-q12 region suggested the ACCN1 gene as the most interesting gene, as the acid-sensing ion channels (ASICs) may be involved in anxiety-related pathways. ${ }^{25-27}$ The ACCN1 gene comprises a genomic region of approximately $1.1 \mathrm{Mb}$ and contains 10 coding exons. According to HapMap (http://hapmap.ncbi.nlm.nih.gov/), approximately 
500 tag-SNPs are required to capture the genetic variation of this gene. However, we selected 55 tag-SNPs, covering exons, exon-intron boundaries, and $3^{\prime}$ and $5^{\prime}$ flanking regions, on the basis of the publicly available genotype data from the Centre $d^{\prime}$ Etude du Polymorphisme Humain (CEPH) trios (http://www.cephb.fr/en/cephdb/), available in the HapMap project dataset (phase II data freeze, assembly NCBI b36, dbSNP b126). The tagging procedure was performed in Haploview, version 3.32. ${ }^{28}$ Two SNPs (rs28936 and rs62068265) were specifically selected to be included due to our positive findings in stage 2 , whereas rs28935 was tagged by rs8066566 $\left(\mathrm{D}^{\prime}=1, r^{2}=1\right)$. The SNPs were genotyped in the extended Faroese sample, and additionally, in the Danish sample. The genotyping was performed using the Sequenom platform as described by Nyegaard et al. ${ }^{20}$ One of the specifically selected SNPs (rs28936) failed to be genotyped. Rs28936 is in very high linkage disequilibrium (LD) with $\operatorname{rs} 28933\left(\mathrm{D}^{\prime}=1, r^{2}=0.91\right)$, which subsequently was analysed using allele-specific hybridization in the LightCycler 480 System (Roche Applied Science, Mannheim, Germany). Primers for rs 28933 were designed and obtained from Tib molbiol (http://www.tib-molbiol.de/de/) (primer sequences are available on request). Quality assurance was achieved by the inclusion of two CEPH controls, which were genotyped for all the SNPs in this study, and showed a $100 \%$ concordance rate with the HapMap genotypes (http://hapmap.ncbi.nlm.nih.gov/). Furthermore, all genotypes were double checked by two researchers.

\section{Statistical analyses}

Quality control. All markers were tested for deviation from HardyWeinberg Equilibrium (HWE), using Exact HWE as implemented in PLINK, ${ }^{29}$ and discarded if the $P$-value was below 0.0001 . Additionally, markers with a call rate below $80 \%$ or a minor allele frequency (MAF) below 0.005 were excluded from further analyses. Furthermore, individuals with missing genotype rate above $10 \%$ were excluded from the analyses.

Stages 1 and 2. Genotypes from the genome-wide scan and the fine mapping of the 17q11.2-q12 region were tested for association using Monte Carlo-based tests as implemented in CLUMP. ${ }^{30}$ The CLUMP statistics presented in this paper are from the subtests $\mathrm{T} 1$ and $\mathrm{T} 4$. The $\mathrm{T} 1$ value is the standard Pearson $\chi^{2}$-test on the raw $2 \times \mathrm{N}$ contingency table, and the T4 value is obtained by reshuffling the columns of the raw $2 \times \mathrm{N}$ table into new $2 \times 2$ tables, until the $\chi^{2}$-value has reached a maximum. Significance level of 0.05 was chosen, however, to compensate for the lack of correction for multiple testing, and to ensure that the applied tests are not too conservative, thresholds of 0.005 for single-locus analysis and 0.01 for two-locus analysis were applied. These different thresholds were chosen mostly based on the different number of alleles/haplotypes analysed in the single-locus and twolocus analyses, and to prevent that tests are too conservative. Alleles and haplotypes of markers with $P$-values below the threshold were, furthermore, tested with Fisher's exact test to detect if specific alleles or haplotypes preferentially displayed a significant skewed distribution. $\mathrm{IBD}_{0}$ was calculated by use of the formula given by Houwen et $a .^{31} \mathrm{IBD}_{0}$ indicates the probability that the given haplotype/ segment is inherited by chance with its observed frequency among the cases, which are related to a known ancestor through a specific genealogical relationship - the 13 cases from the genome-wide scan share a known ancestor living 6.5 generations ago. ${ }^{22}$

Population structure analyses. Analyses of the level of relatedness and inbreeding in the Faroese sample from stage 1 and 2 were based on genome-wide multi locus data from 78 unlinked markers from the genome-wide scan. Genetic differentiation among cases and controls were evaluated by Wright's F-statistic as implemented in SPAGEDi $1.2,{ }^{32}$ and inter- and intra-individual correlations were estimated to evaluate any further genetic subclustering. Furthermore, Bayesian model-based clustering as implemented in STRUCTURE, ${ }^{33}$ was applied to infer any potential cryptic substructure. Models with and without admixture were applied without using any prior information on population structure (ie, disease status).

Stage 3: Amiloride-sensitive cation channel 1 (ACCN1). Allelic association for SNPs located within ACCN1 was tested using the Cochran-Armitage trend test for the Faroese and Danish samples. The Cochran-Mantel-Haenszel $(\mathrm{CMH})$ test was used for the combined Danish and Faroese sample in the open-source software PLINK. ${ }^{29}$ Haplotype association was performed using a sliding window approach of two- and three-marker haplotypes. We report the nominal significant associations, that is, $P$-values below 0.05 . However, none of these SNPs would withstand Bonferroni correcting for multiple testing, which would require a $P$-value below 0.001 . Association at the level of the whole gene or parts of the gene was assessed using the program COMBASSOC performed with 9999 permutations. ${ }^{34}$ COMBASSOC provides a single measurement of significance by combining the $P$-values from all the SNP analyses, and subsequently by permutation testing assessing the empirical significance of the combined $P$-value.

Imputation. To infer missing genotypes and increase genomic coverage, SNP genotypes within the chromosome 17:28 363 219-29509938 region around $A C C N 1$ were imputed using $\mathrm{MaCH} 1.0^{35}$ and the 1000 Genomes maps (August 2009; ftp://share.sph.umich.edu/1000genomes/ pilot1/) as reference haplotypes (CEPH population). Prior to the imputation analysis, five A/T or G/C SNPs and seven SNPs, which were not genotyped in the 1000 Genome project, were excluded. Thus, the imputation analysis was performed using 38 SNPs. Imputed markers with a squared correlation (rsq) between imputed and true genotypes above 0.3 and a quality score above 0.9 were accepted for further analyses. The imputed markers were subsequently analysed for association (trend test and CMH test) using PLINK. ${ }^{29}$

\section{RESULTS}

\section{Stage 1: The genome-wide scan}

In the genome-wide scan, 460 microsatellite markers were successfully genotyped. Several chromosomal regions (4p16.1, 17q11.2-q12 and 19p13.2) showed significant association with PD and increased haplotype sharing among the 13 Faroese patients. The present paper only reports the association between PD and markers on chromosome 17. Using a threshold of 0.01 , we detected a significant association between PD and a two-marker segment (D17S1294-D17S1293) located in the q11.2-q12 region on chromosome 17 (T1: $P$-value $=0.002$; T4: $P$-value $=0.003$ ).

\section{Analyses of genetic relatedness and population structure}

In summary, Wright's F-statistics revealed no signs of genetic differentiation between the 13 cases and 43 controls in the initial Faroese sample. The cluster analysis found it more likely that the data belonged to a single cluster than to multiple clusters. Average withinand between-group kinship coefficients did not differ significantly, indicating that the cases are not closely related to each other than they are to the controls. In conclusion, we observed no significant stratification or cryptic relatedness, and the samples might thus be considered sub-samples with the same genetic background. 
Table 1 Alleles and segments in 17q11.2-q12 showing significant association with PD (stage 2)

\begin{tabular}{|c|c|c|c|c|c|c|c|c|c|c|c|c|c|}
\hline \multirow[b]{3}{*}{ Marker } & \multicolumn{7}{|c|}{ CLUMP } & \multicolumn{4}{|c|}{ Fisherśs exact test } & \multicolumn{2}{|c|}{ IBD by chance } \\
\hline & \multicolumn{3}{|c|}{ Single marker } & \multicolumn{4}{|c|}{ Two markers } & \multirow[b]{2}{*}{ Segment } & \multirow[b]{2}{*}{ Cases } & \multirow[b]{2}{*}{ Controls } & \multirow[b]{2}{*}{$\mathrm{P}$} & \multirow[b]{2}{*}{$(P) I B D_{O}$} & \multirow[b]{2}{*}{ (P) $I B D_{O-\text { sum }}$} \\
\hline & Cases & Controls & $T 1$ & T4 & Marker & $T 1$ & $T 4$ & & & & & & \\
\hline \multicolumn{14}{|l|}{ rs28936 } \\
\hline G & $15(0.58)$ & $22(0.26)$ & 0.0030 & 0.0030 & rs28936-rs28935 & 0.0025 & 0.0028 & & & & & & \\
\hline A & $11(0.42)$ & $62(0.74)$ & & & & & & & & & & & \\
\hline \multicolumn{14}{|c|}{ rs28935 } \\
\hline$A$ & $15(0.58)$ & $74(0.86)$ & 0.0010 & 0.0010 & rs28935-rs62068265 & 0.0033 & 0.0033 & rs28935-rs62068265 & $9 / 26$ & $12 / 86$ & 0.0410 & $2.587 \times 10^{-11}$ & $5.493 \times 10^{-9}$ \\
\hline G & $11(0.42)$ & $12(0.14)$ & & & & & & & & & & & \\
\hline \multicolumn{14}{|c|}{ rs62068265 } \\
\hline G & $24(0.92)$ & $84(0.98)$ & 0.1400 & 0.0140 & rs62068265-rs28933 & 0.0006 & 0.0002 & rs62068265-rs28933 & $15 / 26$ & $18 / 84$ & 0.0011 & $1.230 \times 10^{-22}$ & $2.673 \times 10^{-19}$ \\
\hline C & $2(0.08)$ & $2(0.02)$ & & & & & & & & & & & \\
\hline \multicolumn{14}{|c|}{ rs28933 } \\
\hline A & $15(0.58)$ & $18(0.21)$ & 0.0010 & 0.0010 & rs28933-D17S1540 & 0.0007 & 0.0008 & rs28933-D17S1540 & $8 / 18$ & $4 / 72$ & 0.0002 & $3.521 \times 10^{-11}$ & $5.615 \times 10^{-9}$ \\
\hline G & $11(0.42)$ & $66(0.79)$ & & & & & & & & & & & \\
\hline \multicolumn{14}{|c|}{ D17S1540 } \\
\hline \multirow[t]{2}{*}{ a } & $9(0.35)$ & $9(0.11)$ & 0.0180 & 0.0200 & & & & & & & & & \\
\hline & $17(0.65)$ & $71(0.89)$ & & & & & & & & & & & \\
\hline \multicolumn{14}{|c|}{ D17S1293 } \\
\hline \multirow[t]{2}{*}{ a } & $7(0.27)$ & $9(0.10)$ & 0.1480 & 0.1300 & D17S1293-D17S1842 & 0.0070 & 0.0070 & D17S1293-D17S1842 & $4 / 18$ & $1 / 86$ & 0.0030 & $1.179 \times 10^{-4}$ & $1.03 \times 10^{-2}$ \\
\hline & $19(0.63)$ & $77(0.90)$ & & & & & & & & & & & \\
\hline \multicolumn{14}{|c|}{$D 17 S 1842$} \\
\hline \multirow[t]{3}{*}{ a } & $16(0.62)$ & $60(0.70)$ & 0.0670 & 0.0590 & & & & rs28936-rs28935-rs62068265 & $8 / 20$ & 9/76 & 0.0069 & $9.940 \times 10^{-11}$ & $1.541 \times 10^{-8}$ \\
\hline & $10(0.48)$ & $26(030)$ & & & & & & rs28935-rs62068265-rs28933 & $8 / 18$ & $5 / 78$ & 0.0003 & $3.521 \times 10^{-11}$ & $5.501 \times 10^{-9}$ \\
\hline & & & & & & & & rs62068265-rs28933-D17S1540 & $8 / 20$ & $4 / 70$ & 0.0005 & $9.940 \times 10^{-11}$ & $1.541 \times 10^{-8}$ \\
\hline
\end{tabular}

Empirical CLUMP (T1 and T4 test statistics) for single- and two-marker analyses.

Fisher's exact test for specific haplotypes/segments (alleles not shown).

$\mathrm{IBD}_{0 \text {-sum }}$ shows the same probability summed over all 29 markers in 17q11.2-q12.

aThe allele frequency is given for the allele showing the most skewed distribution against all other alleles.

\section{Stage 2: The 17q11.2-q12 region}

Twenty-two microsatellites and seven SNPs within 17q11.2-q12 were successfully genotyped. The CLUMP analyses showed a significant haplotypic association between the two-locus segment D17S1293D17S1842 and PD, using a threshold of 0.01 (T1: $P$-value $=0.007$; T4: $P$-value $=0.007)$. Fisher's exact test confirmed an overrepresentation of one particular haplotype $(P$-value $=0.003$; Table 1$)$. No significant association was observed between the 5-HTTLPR repeat within the promoter region of the SLC6A4 gene and PD. Single marker analysis of SNPs within ACCN1 revealed a significant association between PD and three SNPs, using a threshold of $0.005, P$-values ranging from 0.001 to 0.003 (Table 1). The same three SNPs and an adjacent distal microsatellite marker D17S1540 displayed, in addition, significant association in the two-marker analyses performed using CLUMP and using a threshold of $0.01, P$-values ranging from 0.003 to 0.0002 (Table 1 ). It appears very unlikely that the observed segments in the case group are inherited IBD by chance through the known genealogy (Table 1).

Stage 3: Amiloride-sensitive cation channel 1 (ACCN1)

A total of 50 SNPs were successfully genotyped with an average call rate of 0.98 (one SNP was monomorphic and five SNPs failed to be genotyped). The statistical analyses in stage 3 were performed separately for the three samples: the Faroese case-control samples, the Danish case-control sample and the combined sample of Danish and Faroese cases versus Danish and Faroese controls. None of the SNPs showed significant deviation from HWE in the three samples of controls. One SNP (rs11868226) was excluded due to frequency test (MAF) in the Faroese sample, whereas none were excluded in the Danish and combined Faroese and Danish samples. Nineteen of 193 individuals ( 2 cases and 17 controls) in the Faroese sample, and 18 of 888 individuals ( 3 cases and 15 controls) in the Danish sample were removed due to low genotyping. No significant association at the level of the whole gene was detected in any of the three samples, neither when including all ten exons, nor when dividing the gene into smaller parts.

The Faroese sample. Six SNPs showed nominal significant allelic association with $\mathrm{PD}$ in the trend test, $P$-values ranging from 0.016 to 0.044 (Table 2). Furthermore, the haplotype analysis showed nominal significant association between PD and several haplotypes, $P$-values ranging from 0.009 to 0.047 for the two-marker haplotypes, and 0.0064 to 0.041 for the three-marker haplotypes (data not shown). Several of the SNPs included in the significantly associated haplotypes were furthermore allelic associated.

The Danish samples. The association analysis showed one SNP nominally significantly allelic associated with PD (rs9915774; Pvalue $=0.031) \quad($ Table 2 ). The haplotype analyses revealed several two- and three-marker haplotypes nominal significantly associated with PD, $P$-values ranging from 0.003 to 0.046 (data not shown). Rs9915774 was furthermore included in the significantly associated two- and three-marker haplotypes. 
Table 2 Significantly associated SNPs within ACCN1 analysed in the extended Faroese (FO) and Danish (DK) case-control samples and in the combined sample between Faroese and Danish cases versus Faroese and Danish controls (FO+DK) (stage 3)

\begin{tabular}{|c|c|c|c|c|c|c|c|c|c|}
\hline \multirow[b]{2}{*}{ SNP } & \multicolumn{3}{|c|}{$F O$} & \multicolumn{3}{|c|}{$D K$} & \multicolumn{3}{|c|}{$F O+D K$} \\
\hline & Cases & Controls & $\mathrm{P}_{\text {trend }}$ & Cases & Controls & $\mathrm{P}_{\text {trend }}$ & Cases & Controls & $\mathrm{P}$ \\
\hline \multicolumn{10}{|c|}{ RS8066566 } \\
\hline A & $16(0.28)$ & $41(0.14)$ & 0.016 & $62(0.13)$ & $192(0.15)$ & 0.245 & $78(0.15)$ & $233(0.15)$ & 0.805 \\
\hline G & $42(0.72)$ & $245(0.86)$ & & $414(0.87)$ & $1066(0.85)$ & & $456(0.85)$ & $1311(0.85)$ & \\
\hline \multicolumn{10}{|c|}{ RS16589 } \\
\hline$A$ & $10(0.17)$ & $93(0.32)$ & 0.020 & $164(0.34)$ & $434(0.34)$ & 0.895 & $174(0.32)$ & $527(0.34)$ & 0.392 \\
\hline G & $48(0.83)$ & $195(0.68)$ & & $316(0.66)$ & $824(0.66)$ & & $364(0.68)$ & $1019(0.66)$ & \\
\hline \multicolumn{10}{|c|}{$R S 16585$} \\
\hline $\mathrm{G}$ & $2(0.03)$ & $45(0.16)$ & 0.016 & $47(0.10)$ & $145(0.12)$ & 0.297 & 49 (0.09) & $190(0.12)$ & 0.072 \\
\hline$A$ & $56(0.97)$ & $245(0.84)$ & & $433(0.90)$ & $1115(0.88)$ & & $489(0.91)$ & $1360(0.88)$ & \\
\hline \multicolumn{10}{|c|}{$R S 12451625$} \\
\hline$A$ & $1(0.02)$ & $28(0.10)$ & 0.044 & $37(0.08)$ & $102(0.08)$ & 0.784 & $38(0.07)$ & $130(0.08)$ & 0.354 \\
\hline G & $57(0.98)$ & $262(0.90)$ & & $443(0.92)$ & $1158(0.92)$ & & $500(0.93)$ & $1420(0.92)$ & \\
\hline \multicolumn{10}{|c|}{$R S 4289044$} \\
\hline$G$ & $17(0.29)$ & $47(0.16)$ & 0.026 & $116(0.24)$ & $278(0.22)$ & 0.346 & $133(0.25)$ & $325(0.21)$ & 0.094 \\
\hline C & $41(0.71)$ & $243(0.84)$ & & $362(0.76)$ & $980(0.78)$ & & $403(0.75)$ & $1223(0.79)$ & \\
\hline \multicolumn{10}{|c|}{ RS8070997 } \\
\hline $\mathrm{G}$ & $7(0.12)$ & $12(0.04)$ & 0.018 & $78(0.16)$ & $175(0.14)$ & 0.229 & $85(0.16)$ & $187(0.12)$ & 0.069 \\
\hline$A$ & $51(0.88)$ & $278(0.96)$ & & $402(0.84)$ & $1085(0.86)$ & & $453(0.84)$ & $1363(0.88)$ & \\
\hline \multicolumn{10}{|c|}{ RS9915774 } \\
\hline$A$ & $5(0.09)$ & $53(0.19)$ & 0.072 & $58(0.12)$ & $204(0.16)$ & 0.033 & $63(0.12)$ & $257(0.17)$ & 0.006 \\
\hline $\mathrm{G}$ & $53(0.91)$ & $233(0.81)$ & & $422(0.88)$ & $1054(0.84)$ & & $475(0.88)$ & $1287(0.83)$ & \\
\hline
\end{tabular}

The allele counts are given in numbers and the frequencies are shown in brackets.

The combined Faroese and Danish sample. Nominal significant allelic association was observed between $\mathrm{PD}$ and $\mathrm{rs} 9915774$ ( $P$-value $=0.007$; Table 2). The haplotype analyses revealed several two- and threemarker haplotypes nominal significantly associated with PD, $P$-values ranging from 0.006 to 0.042 (data not shown). Rs9915774 was one of the SNPs in the significantly associated haplotypes.

\section{Imputation}

In the $1.15-\mathrm{Mb}$ region around $A C C N 1,3786$ SNPs were imputed using 38 SNPs, and 415 of these had an rsq above 0.3 and a quality score above 0.9 . The genotype distribution for the imputed SNPs did not deviate significantly from HWE. In the Faroese sample, the trend test revealed nominal significant allelic association between PD and 69 of the imputed SNPs, including three SNPs genotyped in this study, $P$-values ranging from 0.002 to 0.049 (data not shown). Furthermore, in the Danish sample, the trend test showed nominal allelic association between $\mathrm{PD}$ and 39 of the imputed SNPs, including one of which was genotyped in this study, $P$-values ranging from 0.004 to 0.038 (data not shown). No overlap of nominal significantly associated markers was observed between the Faroese and Danish samples. In the combined Faroese and Danish sample, the $\mathrm{CMH}$ test showed nominal significant allelic association between PD and 39 of the imputed SNPs, including one of the SNPs genotyped in this study, $P$-values ranging from 0.005 to 0.045 (data not shown).

\section{DISCUSSION}

In the search for susceptibility genes for PD, we conducted a genomewide scan (stage 1) and a subsequent fine-scaled follow-up study (stage 2) on PD patients and control individuals from the Faroe Islands. The results revealed significant allelic association and increased haplotype sharing on chromosome 17q11.2-q12, and a possible implication of the ACCN1 gene located within this region. In stage 3, we analysed $A C C N 1$ for association with PD in an extended Faroese case-control sample, using tag-SNPs. Several SNPs within this gene were nominal associated with PD in this extended sample. With the intention of replicating the findings in a larger outbred population, we analysed ACCN1 for association with PD in a Danish sample. The results revealed one significantly associated SNP. The subsequent imputation analyses added no substantial significant association between ACCN1 and PD in any of the samples.

An important issue in mapping susceptibility genes for common complex disorders is whether the genetic factors are likely to be common or rare in a population. Using an isolated population provides increased power to our study to detect rare variants, which increasingly are being identified for common complex disorders. ${ }^{36,37}$ Isolated populations might pose an advantage over outbreed populations in detecting rare variants, ${ }^{38}$ as only a low number of each risk alleles is likely to be introduced into an isolated founder population. In this way, heterogeneity will be reduced, and the LD surrounding the risk variant will be confined to the population history of the isolate. ${ }^{12}$ 
Therefore, rare risk variants identified in isolated populations might not necessarily explain the susceptibility of a disorder or be useful as diagnostic markers in outbred populations. However, they could provide important clues about the mechanism underlying a disorder and thereby contribute to the understanding of the etiology of a disorder like PD. In contrast, isolated populations might not be beneficial in mapping common disease variants of low-effect size. Common alleles would not necessarily be enriched in the isolated population, as multiple founders most likely have introduced the same risk allele into the founder population. ${ }^{39,40}$

The results of this study should be interpreted in the context of several potential limitations. First, we did not correct for multiple testing in stage 1 and 2 using standardised methods. The Bonferroni correction, which assumes independence between the individual tests, was considered too conservative. As many of the markers are in close proximity, they are likely in $\mathrm{LD}^{41}$ and the association tests performed are hence not independent. However, to compensate for multiple testing and reduce the type I error rate, we applied relatively low thresholds in stage 1 and 2. Furthermore, the combined approach with association analysis and IBD estimates should reduce the number of stochastic single-point observations. In stage 3, no correction for multiple testing was performed, and therefore, the nominal-associated observations might represent false positives.

Second, even though we used an isolated population, which may justify the small sample size in the Faroese sample, we cannot ignore that this may affect the $P$-values and the power to detect true difference in allele frequencies between cases and controls. Third, most of the association was confined to the Faroese sample, which might suggest that possible risk alleles genotyped in the present study are not necessarily those involved in the development of PD in a larger outbred population. However, it is possible that low number of founders, isolation and genetic drift followed by rapid exponential population growth has rendered the Faroese population homogenous enough to be able to detect possible risk alleles not detectable in the larger outbred population. To confirm or reject the trend for association observed between markers located within ACCN1 and PD, it might be of interest to analyse Norwegian and Scottish/Irish casecontrol samples, as these populations most likely contributed much more to the founding of the Faroese population than the Danish. ${ }^{14,15}$ Fourth, we did not consider the possible population stratification in the extended Faroese sample and the Danish sample. However, we detected no significant stratification between cases and controls in the initial Faroese sample, which might apply to the extended Faroese sample as well, considering the assumed reduced-genetic heterogeneity in isolated populations. ${ }^{41}$ But, we should not ignore that even apparently homogeneous and isolated populations may have levels of population stratification. ${ }^{42}$ Fifth, we excluded the large intron comprising $1 \mathrm{Mb}$ of the gene, and have therefore not described all the genetic variation within ACCN1. Using the 50 tag-SNPs successfully genotyped in this study, we were able to describe the genetic variation of 120 SNPs. We find this strategy sensible, as 500 tag-SNPs would be required to capture all the genetic variation. The $17 \mathrm{q} 11.2-$ q12 region, comprising a deletion, which recently has been associated with autism spectrum disorder and schizophrenia, ${ }^{43}$ contains other interesting candidate genes, one of which is myosin 1D (MYO1D) previously associated with major autism. ${ }^{44}$ Furthermore, two transmembrane proteins - transmembrane protein 98 (TMEM98) and transmembrane protein 132E (TMEM132E; see Figure 1) - are located in close proximity to $A C C N 1$. Recent studies have shown a possible role of transmembrane gene 132D (TMEM132D) in the etiology of
PD. ${ }^{45,46}$ It might, therefore, be relevant to analyse these genes in future studies of PD.

In summary, we observed nominal association between PD and SNPs within the ACCN1 gene, yet it is unlikely from our data that $A C C N 1$ has a major role in the general genetic susceptibility of PD. We can therefore not confirm the involvement of ASICs in triggering panic attacks. This is consistent with the inconclusive results from association analysis between anxiety spectrum disorders and ACCN2. ${ }^{47}$ It is still unknown whether there are susceptibility genes with major effects in the etiology of PD; therefore, ACCN1 might be one of numerous susceptibility genes for $\mathrm{PD}$, each contributing a moderate effect. Furthermore, most of the association was confined to the Faroese sample, which might be due to the different population history of the study populations. Thus, it might be of interest to analyse ACCN1 in Norwegian and Scottish/Irish case-control samples.

\section{CONFLICT OF INTEREST}

Authors AGW, HAD, OM, TAK declare a potential financial interest in a patent obtained by the Genetic Biobank of the Faroe Islands (Registration number in Denmark: 2137539).

\section{ACKNOWLEDGEMENTS}

This study was supported by grants from the Lundbeck Foundation, the 'Færøske Forskningsråd', the Ivan Nielsen Foundation and the 'Forskningsfond til Støtte af Psykiatrisk Forskning i Region Midtjylland'. Furthermore, we would like to thank the Genetic Biobank of the Faroe Islands for the Faroese sample.

\section{WEB RESOURCES}

$\mathrm{MaCH}$ 1.0: www.sph.umich.edu/csg/abecasis/MACH/tour/ input_files.html

HapMap: http://hapmap.ncbi.nlm.nih.gov/

Tib molbiol: http://www.tib-molbiol.de/de/

Centre d’Etude du Polymorphisme Humain: http://www.cephb.fr/en/cephdb/

1 Kessler RC, Aguilar-Gaxiola S, Alonso J et al: The global burden of mental disorders: an update from the WHO World Mental Health (WMH) surveys. Epidemiol Psichiatr Soc 2009; 18: 23-33.

2 Kessler RC, Chiu WT, Jin R, Ruscio AM, Shear K, Walters EE: The epidemiology of panic attacks, panic disorder, and agoraphobia in the National Comorbidity Survey Replication. Arch Gen Psychiatry 2006; 63: 415-424.

3 Organization WH: The ICD-10 classification of mental and behavioural disorders. Diagnostic Criteria for Research. : World Health Organization, Geneva, 1993.

4 Erickson SR, Guthrie S, Vanetten-Lee M et al: Severity of anxiety and work-related outcomes of patients with anxiety disorders. Depress Anxiety 2009; 26: 1165-1171.

5 Finn CT, Smoller JW: The genetics of panic disorder. Curr Psychiatry Rep 2001; 3: 131-137.

6 Torgersen S: Genetic factors in anxiety disorders. Arch Gen Psychiatry 1983; 40: 1085-1089.

7 Perna G, Caldirola D, Arancio C, Bellodi L: Panic attacks: a twin study. Psychiatry Res 1997; 66: 69-71.

8 Hettema JM, Neale MC, Kendler KS: A review and meta-analysis of the genetic epidemiology of anxiety disorders. Am J Psychiatry 2001; 158: 1568-1578.

9 Klauke B, Deckert J, Reif A, Pauli P, Domschke K: Life events in panic disorder-an update on 'candidate stressors'. Depress Anxiety 2010; 27: 716-730.

10 Fyer AJ, Hamilton SP, Durner $M$ et al: A third-pass genome scan in panic disorder: evidence for multiple susceptibility loci. Biol Psychiatry 2006; 60: 388-401.

11 Kristiansson K, Naukkarinen J, Peltonen L: Isolated populations and complex disease gene identification. Genome Biol 2008; 9: 109.

12 Kruglyak L: Genetic isolates: separate but equal? Proc Natl Acad Sci USA 1999; 96 : 1170-1172.

13 Wright AF, Carothers AD, Pirastu M: Population choice in mapping genes for complex diseases. Nat Genet 1999; 23: 397-404.

14 Als TD, Jorgensen TH, Borglum AD, Petersen PA, Mors O, Wang AG: Highly discrepant proportions of female and male Scandinavian and British Isles ancestry within the isolated population of the Faroe Islands. Eur J Hum Genet 2006; 14: 497-504.

15 Jorgensen TH, Buttenschon HN, Wang AG, Als TD, Borglum AD, Ewald $H$ : The origin of the isolated population of the Faroe Islands investigated using $Y$ chromosomal markers. Hum Genet 2004; 115: 19-28. 
16 Lauritsen MB, Als TD, Dahl HA et al: A genome-wide search for alleles and haplotypes associated with autism and related pervasive developmental disorders on the Faroe Islands. Mol Psychiatry 2006; 11: 37-46.

17 Jorgensen TH, Borglum AD, Mors 0 et al: Search for common haplotypes on chromosome $22 q$ in patients with schizophrenia or bipolar disorder from the Faroe Islands. Am J Med Genet 2002; 114: 245-252.

18 Severinsen JE, Als TD, Binderup $\mathrm{H}$ et al: Association analyses suggest GPR24 as a shared susceptibility gene for bipolar affective disorder and schizophrenia. Am J Med Genet B Neuropsychiatr Genet 2006; 141B: 524-533.

19 Severinsen JE, Bjarkam CR, Kiaer-Larsen S et al: Evidence implicating BRD1 with brain development and susceptibility to both schizophrenia and bipolar affective disorder. Mol Psychiatry 2006; 11: 1126-1138.

20 Nyegaard M, Severinsen JE, Als TD et al: Support of association between BRD1 and both schizophrenia and bipolar affective disorder. Am J Med Genet B Neuropsychiatr Genet 2010; 153B: 582-591.

21 Wing JK, Sturt E: The PSE-ID-CATEGO system: a sup- plementary manual : Institute of Psychiatry, London (mimeo), 1978.

22 Wang AG, Dahl HA, Vang M et al: Genetics of panic disorder on the Faroe Islands: a replication study of chromosome 9 and panic disorder. Psychiatr Genet 2006; 16: 99-104.

23 Koefoed P, Woldbye DP, Hansen TO et al: Gene variations in the cholecystokinin system in patients with panic disorder. Psychiatr Genet 2010; 20: 59-64.

24 Kristensen AS, Mortensen EL, Mors 0: The structure of emotional and cognitive anxiety symptoms. J Anxiety Disord 2009; 23: 600-608.

25 Ziemann AE, Allen JE, Dahdaleh NS et al: The amygdala is a chemosensor that detects carbon dioxide and acidosis to elicit fear behavior. Cell 2009; 139: 1012-1021.

26 Wemmie JA, Askwith CC, Lamani E, Cassell MD, Freeman Jr JH, Welsh MJ: Acidsensing ion channel 1 is localized in brain regions with high synaptic density and contributes to fear conditioning. J Neurosci 2003; 23: 5496-5502.

27 Wemmie JA, Coryell MW, Askwith CC et al: Overexpression of acid-sensing ion channel $1 \mathrm{a}$ in transgenic mice increases acquired fear-related behavior. Proc Natl Acad Sci USA 2004; 101: 3621-3626

28 Barrett JC, Fry B, Maller J, Daly MJ: Haploview: analysis and visualization of LD and haplotype maps. Bioinformatics 2005; 21: 263-265.

29 Purcell S, Neale B, Todd-Brown K et al: PLINK: a tool set for whole-genome association and population-based linkage analyses. Am J Hum Genet 2007; 81: 559-575.

30 Sham PC, Curtis D: Monte Carlo tests for associations between disease and alleles at highly polymorphic loci. Ann Hum Genet 1995; 59: 97-105.

31 Houwen RH, Baharloo S, Blankenship $\mathrm{K}$ et al: Genome screening by searching for shared segments: mapping a gene for benign recurrent intrahepatic cholestasis. Nat Genet 1994; 8: 380-386.
32 Hardy OJ, Vekemans X: SPAGeDi: a versatile computer program to analyse spatial genetic structure at the individual or population levels. Mol Ecol Notes 2002; 2: 618-620.

33 Pritchard JK, Stephens M, Donnelly P: Inference of population structure using multilocus genotype data. Genetics 2000; 155: 945-959.

34 Curtis D, Vine AE, Knight J: A simple method for assessing the strength of evidence for association at the level of the whole gene. Adv Appl Bioinformatics Chem 2008; 1: 115-120.

35 Li Y AG: Mach 1.0: rapid haplotype reconstruction and missing genotype inference. Am J Hum Genet 2006; S79: 2290.

36 Williams NM, Zaharieva I, Martin A et al: Rare chromosomal deletions and duplications in attention-deficit hyperactivity disorder: a genome-wide analysis. Lancet 2010; 376: 1401-1408.

37 Carroll LS, Williams NM, Moskvina $\mathrm{V}$ et al: Evidence for rare and common genetic risk variants for schizophrenia at protein kinase C, alpha. Mol Psychiatry 2010; 15: 1101-1111.

38 Heutink P, Oostra BA: Gene finding in genetically isolated populations. Hum Mol Genet 2002; 11: 2507-2515.

39 Reich DE, Lander ES: On the allelic spectrum of human disease. Trends Genet 2001; 17: $502-510$

40 Lander ES: The new genomics: global views of biology. Science 1996; 274 : 536-539.

41 Jorgensen TH, Degn B, Wang AG et al: Linkage disequilibrium and demographic history of the isolated population of the Faroe Islands. Eur J Hum Genet 2002; 10: 381-387.

42 Helgason A, Yngvadottir B, Hrafnkelsson B, Gulcher J, Stefansson K: An Icelandic example of the impact of population structure on association studies. Nat Genet 2005; 37: 90-95.

43 Moreno-De-Luca D, Mulle JG, Kaminsky EB et al: Deletion 17q12 is a recurrent copy number variant that confers high risk of autism and schizophrenia. Am J Hum Genet 2010; 87: 618-630.

44 Stone JL, Merriman B, Cantor RM, Geschwind DH, Nelson SF: High density SNP association study of a major autism linkage region on chromosome 17. Hum Mol Genet 2007; 16: 704-715.

45 Erhardt A, Czibere L, Roeske D et al: TMEM132D, a new candidate for anxiety phenotypes: evidence from human and mouse studies. Mol Psychiatry 2010.

46 Otowa T, Yoshida E, Sugaya N et al: Genome-wide association study of panic disorder in the Japanese population. J Hum Genet 2009; 54: 122-126.

47 Hettema JM, An SS, Neale MC, van den Oord EJ, Kendler KS, Chen X: Lack of association between the amiloride-sensitive cation channel 2 (ACCN2) gene and anxiety spectrum disorders. Psychiatr Genet 2008; 18: 73-79. 